\title{
MHC class II associated stomach cancer mutations correlate with lack of subsequent tumor development
}

\author{
JOHN M. YAVORSKI ${ }^{1}$ and GEORGE BLANCK ${ }^{1,2}$ \\ ${ }^{1}$ Department of Molecular Medicine, Morsani College of Medicine, University of South Florida, Tampa; \\ ${ }^{2}$ Immunology Program, H. Lee Moffitt Cancer Center and Research Institute, Tampa, FL 33612, USA
}

Received February 9, 2017; Accepted August 17, 2017

DOI: $10.3892 / \mathrm{mco} .2017 .1432$

\begin{abstract}
The role of tumor cell expression of major histocompatibility class II (MHCII) has been controversial, with evidence indicating that tumor cell expression of MHCII may lead to an anti-tumor immune response and to tumor cell apoptosis and that MHCII has pro-tumorigenic functions. The cancer genome atlas (TCGA) indicates numerous deleterious mutations for the highly specific, MHCII transcriptional activation proteins, RFX5, RFXAP, RFXANK and CIITA. Also, mutations in the non-polymorphic, human leukocyte antigen (HLA)-DRA gene, which encodes the heavy chain for the most prominent human MHCII molecule, HLA-DR, are common. For many, if not most TCGA cancer datasets, the MHCII specific mutations do not associate with clinical outcomes. However, stomach carcinoma represents an exception, where the data indicate that MHCII-specific mutations are associated with a more favorable outcome. These data raise the question of whether stomach cancer mutations represent effective haploinsufficiency or whether mutations that are associated with a favorable outcome occur with other stomach cancer molecular features that limit the function of the two alleles that represent these MHCII-related proteins.
\end{abstract}

\section{Introduction}

The potential impact of MHCII expression on solid tumor cells received increased interest when it became apparent over two

Correspondence to: Professor George Blanck, Department of Molecular Medicine, Morsani College of Medicine, University of South Florida, 12901 Bruce B. Downs Bd. MDC7, Tampa, FL 33612, USA

E-mail: gblanck@health.usf.edu

Abbreviations: MHC, major histocompatibility complex; STAD, stomach adenocarcinoma; TCGA, the cancer genome atlas; CIITA, class II transactivator; RFX, regulatory factor-X; SKCM, skin cutaneous melanoma; LUAD, lung adenocarcinoma; COAD, colon adenocarcinoma; BLCA, bladder carcinoma; HNSC, head and neck cancer

Key words: major histocompatibility class II proteins, HLA-DR, solid tumor, interferon- $\gamma$ induction, stomach cancer, prognosis decades ago that mutations specific to tumorigenesis interfered with MHCII induction by interferon- $\gamma($ IFN- $\gamma$ ) (1-9). As one example, a lack of retinoblastoma tumor suppressor protein leads to over expression of the pro-proliferative protein, YY1, which in turn is part of a repressive complex that maintains histone deacetylase activity at the MHCII promoter, thereby blocking the assembly of MHCII enhanceosome proteins, including the highly specific MHCII enhanceosome proteins, RFXANK, RFXAP, RFX5 and CIITA $(10,11)$. In addition, Ostrand-Rosenberg and colleagues $(12,13)$ have established the negative impact of tumor cell-MHCII expression on tumor development, although there remain questions about whether such a negative impact occurs in a natural state, where there is the expectation of CLIP expression blocking endogenous MHCII tumor-peptide loading, or in the absence of tumor cell expression of conventional co-stimulatory molecules. The apoptotic mechanisms of tumor cell MHCII expression provide another possible 'anti-tumor' role $(14,15)$.

\section{Data collection methods}

Clinical and primary tumor specimen mutation Microsoft Excel files for the stomach adenocarcinoma (STAD), skin cutaneous melanoma (SKCM), lung adenocarcinoma (LUAD), colon adenocarcinoma (COAD), head and neck squamous cell carcinoma (HNSC) and bladder urothelial carcinoma (BLCA) cancer sets was downloaded from the TCGA data portal (dbGaP project approval number 6300). The 'new tumor event after initial treatment' column of the TCGA clinical follow up file for each cancer dataset was used to categorize barcodes based on the development of a new tumor or not (Table I, New Tumor and No Subsequent Tumor, respectively). To obtain matching barcodes, for the clinical and somatic mutation files, the barcodes from the primary tumor specimen mutation file were truncated to contain the following characters, TCGA-\#\#-\#\#\#\#. Mutation data, including truncated tumor sample barcodes, human genome organization symbols and mutation type (nonsynonymous or silent) for HLA-DRA and the set of transcription factors (CIITA, RFX5, RFXANK and RFXAP) associated with MHC Class II were collected for each cancer dataset. Mutations were assessed using the PROVEAN web tool (16) The Excel COUNTIF function was used to obtain the number of MHC Class II coding region mutations per barcode for the New Tumor and No Subsequent Tumor 
Table I. MHC class II associated mutation data for the six TCGA cancer datasets studied.

\begin{tabular}{|c|c|c|c|c|c|c|}
\hline & \multicolumn{6}{|c|}{ TCGA cancer dataset } \\
\hline & STAD & SKCM & LUAD & COAD & HNSC & BLCA \\
\hline Number of MHC class II coding region mutations from TCGA & 47 & 106 & 42 & 39 & 33 & 33 \\
\hline Number of coding region mutations when silent mutations removed & 39 & 65 & 34 & 29 & 25 & 24 \\
\hline Total sample size from TCGA & 379 & 470 & 542 & 216 & 510 & 395 \\
\hline Number of CIITA mutations & 16 & 37 & 7 & 8 & 15 & 9 \\
\hline Number of RFX5 mutations & 13 & 6 & 23 & 13 & 5 & 7 \\
\hline Number of RFXANK mutations & 3 & 2 & 1 & 1 & 0 & 1 \\
\hline Number of RFXAP mutations & 1 & 2 & 0 & 2 & 3 & 2 \\
\hline Number of HLA-DRA mutations & 6 & 18 & 3 & 5 & 2 & 5 \\
\hline Total mutations & 39 & 65 & 34 & 29 & 25 & 24 \\
\hline Number of New Tumor barcodes available for study & 74 & 101 & 166 & 48 & 63 & 82 \\
\hline Number of No Subsequent Tumor barcodes available for study & 224 & 182 & 243 & 156 & 215 & 146 \\
\hline
\end{tabular}

Data compiled from SOM file labeled, 'SOM, MHC Class II' available at http://universityseminarassociates.com/media/SOM_MHC_Class_ II.pdf. The mutation totals for the five coding regions represent non-synonymous mutations, i.e., silent mutations removed. STAD, stomach adenocarcinoma; SKCM, skin cutaneous melanoma; LUAD, lung adenocarcinoma; COAD, colon adenocarcinoma; HNSC, head and neck squamous cell carcinoma; BLAC, bladder urothelial carcinoma; HLA, human leukocyte antigen; MHC, major histocompatibility complex; TCGA, The Cancer Genome Atlas.

Table II. The average number of mutations per barcode and statistical comparison of the New Tumor and No Subsequent Tumor sets

\begin{tabular}{llrrrrr}
\hline & & \multicolumn{4}{c}{ TCGA cancer dataset } \\
\cline { 2 - 6 } & STAD & SKCM & LUAD & COAD & HNSC & BLCA \\
\hline Avg. mutations per barcode for New Tumor group & 0.027 & 0.178 & 0.084 & 0.188 & 0.016 & 0.037 \\
Avg. mutations per barcode for No Subsequent Tumor group & 0.138 & 0.137 & 0.062 & 0.109 & 0.042 & 0.110 \\
P-value comparison of New Tumor and No Subsequent Tumor & 0.004 & 0.512 & 0.481 & 0.275 & 0.238 & 0.052 \\
\hline
\end{tabular}

Data compiled from SOM file labeled, 'SOM, MHC Class II' available at http://universityseminarassociates.com/media/SOM_MHC_Class_ II.pdf. Avg, average; STAD, stomach adenocarcinoma; SKCM, skin cutaneous melanoma; LUAD, lung adenocarcinoma; COAD, colon adenocarcinoma; HNSC, head and neck squamous cell carcinoma; BLAC, bladder urothelial carcinoma; TCGA, The Cancer Genome Atlas.

groups for each cancer dataset and a statistical comparison between the groups was conducted. t-tests were used to obtain $\mathrm{P}$-values. $\mathrm{P}<0.05$ was considered to indicate a statistically significant difference. P-values were obtained using Microsoft Excel (Microsoft Corporation, Redmond, WA, USA).

All procedures performed in studies involving human participants were in accordance with the ethical standards of the institutional and national research committee and with the 1964 Helsinki declaration and its later amendments or comparable ethical standards. The present study is exempt from IRB approval and was approved via the National Institutes of Health, Database of Phenotypes and Genotypes (dbGaP), project no. 6,300; approval granted to George Blanck.

\section{Results and discussion}

TCGA provides a wealth of information regarding mutagenesis in many cancer datasets. To obtain an indication of mutations that may impact MHCII expression, TCGA was searched for mutations in RFXAP, RFXANK, RFX5, CIITA and HLA-DRA. Other MHCII structural genes were excluded due to the potential confusion caused by the high level of polymorphisms. Overall, the nonsynonymous mutation rate for the following TCGA datasets, for the above collection of MHCII specific proteins, was 8-9\%: STAD, SKCM, LUAD, COAD, HNSC and BLCA.

The opportunities for linking TCGA clinical information, particularly negative vs. positive outcomes, to particular mutations remains limited, largely owing to the minimal overlap of barcodes (patient samples) for mutation results and clinical information. However, it is possible to attempt to correlate mutation results with either no-subsequent tumor or new-tumor for the above cancer datasets, particularly due to the relatively high number of barcodes available representing this distinction (Table I). Of the six TCGA datasets representing a substantial number of mutations and no-subsequent tumor and new-tumor cases, only the stomach cancer datasets (STAD) demonstrated a correlation with the MHCII specific mutations, 
namely an association of more mutations with no-subsequent tumor (Table II). Of thirty-one mutations that were in the STAD no-subsequent tumor group, 21 were assessable by the PROVEAN (17) web tool, of which 9 were deleterious and 12 were neutral. The two mutations that were in the new-tumor group were assessable by PROVEAN, revealing that 1 was deleterious and 1 was neutral.

As aforementioned, solid tumor cell expression of MHCII has led to contradictory impressions as to whether MHCII facilitates or inhibits solid tumor development. The above data support the former possibility, but no doubt there are a number of circumstances in which the impact of solid tumor expression of MHCII may have varying effects on tumor progression. For example, varied solid tumors may be affected differently by constitutive MHCII expression or MHCII induction by IFN- $\gamma$. Furthermore, MHCII expression may have different impacts at different stages of tumorigenesis. The issue of the variable impacts of immune function spans the consideration of the role of the immune system in tumor development. Immune checkpoint inhibitors have had great positive benefits for at least a subset of patients (18), yet in other settings, evidence indicates that inflammation, particularly chronic inflammation, is associated with tumor development $(19,20)$.

The negative impact of MHCII expression on solid tumor cells may include induction of T-cell anergy (21), due to lack of costimulatory molecules, but a previous study indicates that non-professional antigen presenting cells, including solid tumor cells, are able to employ substitute co-stimulatory molecules such as ICAM1 (22). Another potential explanation for a negative impact of MHCII expression is the possibility that MHCII facilitates T-cell engulfment by solid tumor cells $(23,24)$.

In conclusion, the current study indicates that, at least in certain situations, the expression of MHCII on tumor cells may represent a negative prognosis. Such a conclusion calls into question scenarios where MHCII-based interactions with the immune system would facilitate an anti-tumor immune response.

\section{Acknowledgements}

The authors would like to acknowledge the support of the Anna Valentine Program and the taxpayers of the State of Florida.

\section{References}

1. Lu Y, Boss JM, Hu SX, Xu HJ and Blanck G: Apoptosisindependent retinoblastoma protein rescue of HLA class II messenger RNA IFN-gamma inducibility in non-small cell lung carcinoma cells. Lack of surface class II expression associated with a specific defect in HLA-DRA induction. J Immunol 156 2495-2502, 1996.

2. Blanck G: Components of the IFN-gamma signaling pathway in tumorigenesis. Arch Immunol Ther Exp (Warsz) 50: 151-158, 2002.

3. Xi H, Eason DD, Ghosh D, Dovhey S, Wright KL and Blanck G Co-occupancy of the interferon regulatory element of the class II transactivator (CIITA) type IV promoter by interferon regulatory factors 1 and 2. Oncogene 18: 5889-5903, 1999.

4. Lu Y, Ussery GD, Muncaster MM, Gallie BL and Blanck G Evidence for retinoblastoma protein (RB) dependent and independent IFN-gamma responses: RB coordinately rescues IFN-gamma induction of MHC class II gene transcription in noninducible breast carcinoma cells. Oncogene 9: 1015-1019, 1994.
5. Lu Y, Tschickardt ME, Schmidt BJ and Blanck G: IFN-gamma inducibility of class II transactivator is specifically lacking in human tumour lines: Relevance to retinoblastoma protein rescue of IFN-gamma inducibility of the HLA class II genes. Immunol Cell Biol 75: 325-332, 1997.

6. Xi H, Goodwin B, Shepherd AT and Blanck G: Impaired class II transactivator expression in mice lacking interferon regulatory factor-2. Oncogene 20: 4219-4227, 2001.

7. $\mathrm{Xi} \mathrm{H}$ and Blanck $\mathrm{G}$ : Interferon regulatory factor-2 point mutations in human pancreatic tumors. Int J Cancer 87: 803-808, 2000.

8. Eason DD, Coppola D, Livingston S, Shepherd AT and Blanck G: Loss of MHC class II inducibility in hyperplastic tissue in Rb-defective mice. Cancer Lett 171: 209-214, 2001.

9. Zhu X, Pattenden S and Bremner R: pRB is required for interferon-gamma-induction of the MHC class II abeta gene. Oncogene 18: 4940-4947, 1999.

10. Osborne A, Zhang H, Yang WM, Seto E and Blanck G: Histone deacetylase activity represses gamma interferon-inducible HLA-DR gene expression following the establishment of a DNase I-hypersensitive chromatin conformation. Mol Cell Biol 21: 6495-6506, 2001.

11. Osborne AR, Zhang H, Fejer G, Palubin KM, Niesen MI and Blanck G: Oct-1 maintains an intermediate, stable state of HLA-DRA promoter repression in Rb-defective cells: An Oct-1-containing repressosome that prevents NF-Y binding to the HLA-DRA promoter. J Biol Chem 279: 28911-28919, 2004.

12. Chornoguz O, Gapeev A, O'Neill MC and Ostrand-Rosenberg S: Major histocompatibility complex class II+ invariant chain negative breast cancer cells present unique peptides that activate tumor-specific $\mathrm{T}$ cells from breast cancer patients. Mol Cell Proteomics 11: 1457-1467, 2012.

13. Srivastava MK, Bosch JJ, Wilson AL, Edelman MJ and Ostrand-Rosenberg S: MHC II lung cancer vaccines prime and boost tumor-specific CD4+ T cells that cross-react with multiple histologic subtypes of nonsmall cell lung cancer cells. Int J Cancer 127: 2612-2621, 2010.

14. Truman JP, Choqueux C, Charron D and Mooney N: HLA class II molecule signal transduction leads to either apoptosis or activation via two different pathways. Cell Immunol 172: 149-157, 1996.

15. Le E, Zhang H and Blanck G: CIITA transformation rescues the apoptotic function of MHC class II in melanoma cells. Anticancer Res 25: 3889-3892, 2005.

16. Stoll RJ, Thompson GR, Samy MD and Blanck G: De novo, systemic, deleterious amino acid substitutions are common in large cytoskeleton-related protein coding regions. Biomed Rep 6: 211-216, 2017.

17. Choi Y, Sims GE, Murphy S, Miller JR and Chan AP: Predicting the functional effect of amino acid substitutions and indels. PLoS One 7: e46688, 2012.

18. Yun S, Vincelette ND, Green MR, Wahner Hendrickson AE and Abraham I: Targeting immune checkpoints in unresectable metastatic cutaneous melanoma: A systematic review and meta-analysis of anti-CTLA-4 and anti-PD-1 agents trials. Cancer Med 5: 1481-1491, 2016.

19. Khawar MB, Abbasi MH and Sheikh N: IL-32: A novel pluripotent inflammatory interleukin, towards gastric inflammation, gastric cancer, and chronic rhino sinusitis. Mediators Inflamm 2016: 8413768, 2016.

20. Fornaro R, Caratto M, Caratto E, Caristo G, Fornaro F, Giovinazzo D, Sticchi C, Casaccia M and Andorno E: Colorectal cancer in patients with inflammatory bowel disease: The need for a real surveillance program. Clin Colorectal Cancer 15: 204-212, 2016.

21. Macián F, Im SH, García-Cózar FJ and Rao A: T-cell anergy. Curr Opin Immunol 16: 209-216, 2004.

22. Kohlmeier JE, Chan MA and Benedict SH: Costimulation of naive human CD4 $\mathrm{T}$ cells through intercellular adhesion molecule- 1 promotes differentiation to a memory phenotype that is not strictly the result of multiple rounds of cell division. Immunology 118: 549-558, 2006.

23. Lugini L, Matarrese P, Tinari A, Lozupone F, Federici C, Iessi E, Gentile M, Luciani F, Parmiani G, Rivoltini L, et al: Cannibalism of live lymphocytes by human metastatic but not primary melanoma cells. Cancer Res 66: 3629-3638, 2006.

24. Lloyd MC, Szekeres K, Brown JS and Blanck G: Class II transactivator expression in melanoma cells facilitates T-cell engulfment. Anticancer Res 35: 25-29, 2015. 